\title{
Original Research Prosthesis \\ Antimicrobial action of sodium hypochlorite and castor oil solutions for denture cleaning - in vitro evaluation
}

\section{Marcela Moreira SALLES Viviane de Cássia OLIVEIRA Raphael Freitas de SOUZA Cláudia Helena Lovato da SILVA Helena de Freitas Oliveira PARANHOS}

Universidade de São Paulo - USP, School of Dentistry of Ribeirão Preto, Department of Dental Materials and Prosthetics, Ribeirão Preto, SP, Brazil.

Declaration of Interests: The authors certify that they have no commercial or associative interest that represents a conflict of interest in connection with the manuscript.

Corresponding Author:

Marcela Moreira Salles

E-mail:cela_salles@yahoo.com.br

DOI: 10.1590/1807-3107BOR-2015.vol29.0104

Submitted: Nov 11, 2014

Accepted for publication: May 19, 2015

Last revision: Jul 14, 2015
Abstract: The objective of this in vitro study was to evaluate the antimicrobial action of sodium hypochlorite $(0.25 \%$ and $0.50 \%)$ and $10 \%$ castor oil solutions against specific microorganisms, by counting Colony Forming Units (CFU) of clinically important bacteria and Candida species. Acrylic resin specimens $(\mathrm{n}=320$; Lucitone 550$)$ were obtained from square metal matrices $(10 \times 10 \times 2 \mathrm{~mm})$, sterilized by microwave (650W, for 6 minutes) and contaminated by Staphylococcus aureus, Pseudomonas aeruginosa, Candida albicans, Bacillus subtilis, Escherichia coli, Streptococcus mutans, Enterococcus faecalis and Candida glabrata. The specimens were immersed for 20 minutes in one of the following hygiene solutions $(n=10 /$ each): A $-0.25 \%$ Sodium hypochlorite; B - 0.5\% Sodium hypochlorite; C - 10\% Castor oil solution; and D (Control) - saline. Adhered cells were suspended and inoculated into a selective solid medium $\left(37^{\circ} \mathrm{C}\right.$ for $\left.24 \mathrm{~h}\right)$. The Student's t-test $(\alpha=0.05)$ was performed to compare $\log 10(\mathrm{CFU}+1) / \mathrm{mL}$ between Groups C and D. The results showed that sodium hypochlorite $(0.25 \%$ and $0.5 \%$ ) completely eliminated all detectable microorganisms. The castor oil solution eliminated B. subtilis and reduced counts for other strains. Differences between C and D were significant $(p<0.05)$ for all species except for $E$. faecalis. Both sodium hypochlorite solutions $(0.25 \%$ and $0.5 \%$ ) were effective in eliminating all microorganisms evaluated, and may be useful as cleaning solutions for complete dentures. The castor oil solution provided moderate efficacy and performed differently on the tested species, with the strongest effect on B. subtilis and with non-significant action on E. faecalis.

Keywords: Biofilms; Denture Cleansers; Sodium Hypochlorite; Ricinus communis; Denture Bases.

\section{Introduction}

Despite the widely recommended act of brushing as an efficacious method to remove denture biofilm, this method depends mostly on manual ability, and may have limited efficacy in some cases. ${ }^{1}$ A way to compensate this is to use a method associating brushing and immersion in solutions; this has been recommended as an effective hygiene procedure. ${ }^{2,3}$ Thus, immersion in chemical solutions has been considered a feasible alternative for denture wearers who need auxiliary cleaning materials. 
Alkaline hypochlorite solutions have demonstrated favorable results for denture hygiene. They act in the organic matrix of the biofilm, present fungicidal and bactericidal properties and can remove stains. ${ }^{3,4}$ The recommendation to use diluted sodium hypochlorite $(\mathrm{NaOCl})$, such as household bleaching solutions, is common. ${ }^{5,6}$ However, these solutions have not only bad taste, ${ }^{6}$ but, most importantly, may damage denture materials, depending on the immersion time and concentration. For instance, they may whiten acrylic resins $^{7}$ and cause corrosion to metal components. ${ }^{8,9}$ Studies have reported the antimicrobial action of $\mathrm{NaOCl}$ in concentrations of $1 \%$ to $5.25 \%,^{10,11,12}$ however, there is no consensus for the most efficacious concentrations and the duration of immersion. Hence, the antimicrobial action of the solution must be evaluated when recommending this product for daily denture hygiene.

It is important to continuously improve existing hygiene methods as well as analyze new formulations to ensure no damage to the denture materials, effectiveness and low cost. In dentistry, compounds made from castor beans (Ricinus communis) have been used, because of their biocompatibility and anti-inflammatory activity, as well as their bactericide and fungicide action. A study evaluated a 3.3\% castor oil detergent and showed antimicrobial activity against anaerobes and streptococci, both present in pulp necrosis. ${ }^{13}$ Another study reported the effectiveness of a $10 \%$ castor oil detergent on the irrigation of root canals, ${ }^{14}$ showing the action of the product on microorganisms present in endodontic infections.

A castor oil-based solution has features favoring its use for denture hygiene, based on its potent detergent and antimicrobial action; in addition, it is colorless and has no unpleasant odor. R. communis is cultivated in several countries, thus making it available to complete dentures users in the future. However, few studies have been conducted regarding the efficacy of castor oil products as complete denture cleansers. In relation to adverse effects, previous studies evaluated a $2 \%$ solution and showed non-significant changes in surface roughness, hardness and color of artificial teeth and acrylic resin..$^{15,16}$ Clinical trials showed that a $2 \%$ castor oil immersion solution was effective in denture biofilm removal, compared with an alkaline peroxide solution, but its antimicrobial action was not evaluated, ${ }^{3}$ and a $3.3 \%$ castor oil mouthwash reduced the clinical signs of denture stomatitis; however, this treatment did not significantly reduce the CFU of Candida spp. ${ }^{17}$

For this reason, a solution with a $10 \%$ castor oil concentration was selected because of its effectiveness against microorganisms present in endodontic infections. ${ }^{14}$ Leite et al. ${ }^{18}$ showed that experimental dentifrice containing $10 \% R$. communis oil was effective against $S$. mutans, $S$. aureus and E. faecalis. Moreover, the literature has not shown adverse effects in a $10 \%$ concentration. Therefore, it is crucial to evaluate the antimicrobial action of these solutions, when applied in immersions of short duration and low concentrations.

The aim of this study was to evaluate the in vitro antimicrobial action of $\mathrm{NaOCl}(0.25$ and $0.50 \%)$ and castor oil (10\%) solutions against bacterial species and Candida spp. The null hypothesis of the study was that the tested immersion solutions would have the same effect against the tested microorganisms.

\section{Methodology}

Square metallic matrices (10 $\mathrm{mm} \times 2 \mathrm{~mm})$ were invested in type III dental stone (Gesso Rio, Rio Claro, Brazil) and putty condensation silicone (Zetalabor; Zermack, Badia Polesine, Italy), in metallic flasks (Jon, São Paulo, Brazil). After the removal of the matrices, heat-polymerized acrylic resin (Lucitone 550; Dentsply Ind. Com. Ltda., Petrópolis, Brazil) was manipulated, packed and pressed into the dental cast at $1200 \mathrm{kgf}$ for $30 \mathrm{~min}$ (Protecni Hydraulic Press, Araraquara, Brazil). The specimens were polymerized by a conventional heating method, according to the manufacturer's instructions (water immersion at $73^{\circ} \mathrm{C}$ for $90 \mathrm{~min}$ and boiling for $30 \mathrm{~min}$ ), in an electric thermopolymerizing device (Thermocycler T100, Ribeirão Preto, Brazil). The specimens were then deflasked ${ }^{8}$ and immersed in distilled water at $50^{\circ} \mathrm{C}$ for $24 \mathrm{~h}$ to eliminate the residual monomer. The excess of polymerized resin was trimmed with a bur (Maxi-Cut, Maillefer SA, Ballaigues, Switzerland), using a low speed micromotor (Dabi Atlante, Ribeirão Preto, Brazil), and the surfaces were polished using 400- and 600-grit wet/dry sandpapers (Norton, Guarulhos, Brazil), in a horizontal lathe spindle (Arotec, Cotia, Brazil). Three hundred and twenty specimens were 
obtained. The specimens were immersed in distilled water and sterilized with microwave irradiation (Panasonic, Kadoma, Japan), Model Perfect, 127V; $800 \mathrm{~W} ; 2450 \mathrm{MHz}$, at $650 \mathrm{~W}$, for $6 \mathrm{~min}^{19}$

An evaluation was made of the efficacy of the hygiene methods against 8 strains from the American Type Culture Collection (ATCC): Staphylococcus aureus (ATCC 25923), Streptococcus mutans (ATCC 25175), Candida albicans (ATCC 10231), Candida glabrata (ATCC 2001), Bacillus subtilis (ATCC 6633), Escherichia coli (ATCC 25922), Enterococcus faecalis (ATCC 29212) and Pseudomonas aeruginosa (ATCC 27853). These microorganisms have been used for controlling and monitoring the antimicrobial activity of various compounds; moreover, these species have been isolated from the oral cavity and dental prosthesis surfaces. . $^{2,12,20}$

Microorganisms were added to saline solution to standardize the selected inoculum. The turbidity of the microbial suspension was verified by spectrophotometer, according to the McFarland scale, and had an absorbance reading from 0.08 to 0.1 , at a wavelength of $625 \mathrm{~nm}$. According to our calibration curves, this absorbance corresponds to $10^{6} \mathrm{CFU} / \mathrm{mL}$ for yeasts and $10^{8} \mathrm{CFU} / \mathrm{mL}$ for bacteria. The culture medium was then inoculated with $1 \%$ microbial inoculum.

In the laminar flow cabinet (Pachane, Pa 400-ECO, Piracicaba, Brazil), the acrylic resin specimens were distributed in 24-well tissue culture plates (TPP; Trasadingen, Switzerland), and $1 \mathrm{~mL}$ of medium broth containing standardized cell suspension was added to each well. The plates were incubated for $90 \mathrm{~min}$ at $37^{\circ} \mathrm{C}$ at $75 \mathrm{rpm}$ (adhesion period). Afterwards, the surfaces were washed with saline to remove non-adherent microorganisms. Biofilm growth was promoted by adding $1 \mathrm{~mL}$ of appropriate sterile medium broth to each well, after which the plates were incubated at $37^{\circ} \mathrm{C}$, at $75 \mathrm{rpm}$ for $48 \mathrm{~h}$, under aerobic or anaerobic conditions.

The specimens $(n=10)$ were randomly assigned to one of the cleansing solutions being evaluated: (A) $0.25 \% \mathrm{NaOCl}$ (Inject Center, Ribeirão Preto, Brazil); (B) $0.5 \% \mathrm{NaOCl}$ (Inject Center); (C) $10 \%$ castor oil solution (Institute of Chemistry, University of São Paulo, São Carlos, Brazil); (D) control group: 0.85\%
Saline (Sodium chloride P.A., Labsynth - Laboratory Products Ltda., Diadema, Brazil).

After incubation, the specimens were removed from the plate and transferred to a tube containing $5 \mathrm{~mL}$ of one of the cleansing solutions or saline. The tubes were immersed in the incubator under orbital agitation at $75 \mathrm{rpm}$, for $20 \mathrm{~min}$, to assure contact of the solutions with all the surfaces. After this immersion period, each specimen was removed aseptically and washed 3 times with saline to remove residual disinfectants and loosely adherent cells. The specimens were then transferred to a tube containing a liquid culture medium (Letheen Broth; Difco Laboratories Inc., Detroit, USA), and the remaining adherent microorganisms were removed from the treated specimens by sonication (Altsonic, Ribeirão Preto, Brazil), for $20 \mathrm{~min}$.

The resultant suspension was vortexed, the initial suspension $\left(10^{\circ}\right)$ was diluted from $10^{-1}$ to $10^{-3}$ in a sterile saline solution, and aliquots were plated onto sterile Petri dishes containing specific medium. The solid culture media used were: Mueller Hinton Agar (HiMedia Laboratories Pvt. Ltda., Mumbai, India) for $P$. aeruginosa, S. aureus, E. coli and B. subtilis; Sabouraud Dextrose Agar (HiMedia Laboratories Pvt. Ltda., Mumbai, India) for C. albicans and C. glabrata; Mitis Salivarius Agar Base (HiMedia Laboratories Pvt. Ltda., Mumbai, India; added to Bacitracin and $20 \%$ sucrose) for S. mutans; and Tryptone Soya Agar (HiMedia Laboratories Pvt. Ltda., Mumbai, India) for $E$. faecalis. The dishes were then incubated at $37^{\circ} \mathrm{C}$ for $24 \mathrm{~h}$ under aerobic or anaerobic conditions (S. mutans and E. faecalis).

After the incubation period, the number of colonies in each dilution was counted, and the value of CFUs was obtained, based on a dilution providing 1-300 colonies: $\mathrm{CFU} / \mathrm{mL}=$ number of colonies $\times 10^{\mathrm{n}} / \mathrm{q}$, where: $\mathrm{n}=$ absolute value of the dilution $(0,1,2$ or 3$)$, and $\mathrm{q}=$ quantity of plated suspension $(0.05 \mathrm{~mL})$.

Statistical tests were performed using the SPSS17.0 program (SPSS Inc., Chicago, USA). CFU values were converted to $\log 10$. There were several readings that resulted in zero $\mathrm{CFU}$, thus the microbial count data obtained were expressed as $\log (\mathrm{CFU}+1)$. The groups were compared by parametric test for independent samples. Since only 2 groups (C and D) resulted in 
detectable CFUs, we used Student's t-test. All analyses were performed at a 0.05 significance level.

\section{Results}

The results of the $\mathrm{CFU} / \mathrm{mL}$ for groups $\mathrm{A}$ and $\mathrm{B}$ $(\mathrm{NaOCl})$ were zero. Table shows the results for Groups C (10\% castor oil solution) and D (control group).

Average counts for Group C ( $10 \%$ castor oil solution) were significantly lower than those for Group D (control), regardless of the species $(p<0.05)$, except for $E$. faecalis, which was not reduced by castor oil. B. subtilis was undetectable in Group C $(10 \%$ castor oil solution), similar to the result found for Groups A and B.

\section{Discussion}

The results showed that the null hypothesis was rejected, because both $\mathrm{NaOCl}$ solutions $(0.25 \%$ and $0.5 \%$ ) were effective in eliminating all the microorganisms evaluated, and the castor oil solution provided moderate efficacy and performed differently for the species tested.

In this study, the immersions were performed as an isolated method, to obtain an objective evaluation of the antimicrobial action of each solution, since the association of a chemical method with a mechanical method promotes synergistic activity. ${ }^{2,5}$ The study evaluated the effectiveness of the tested solutions against important microorganisms recommended to assess the antimicrobial action of several disinfectant agents. ${ }^{20}$

The following important gram positive bacteria were selected: $S$. mutans was chosen for its role as a primary oral biofilm colonizer and its involvement in the development of caries and gingivitis; $S$. aureus has been associated with infections, such as angular cheilitis, endodontic infections, parotiditis and oral mucositis; ${ }^{21} E$. faecalis has been associated with oral cavity diseases, such as apical periodontitis and endodontic infections; ${ }^{22}$ B. subtilis, a non-pathogenic gram positive bacterium, contributes to biofilm growth and, consequently, to pathogen microorganism adherence. ${ }^{23}$ The following gram negative bacteria were selected: E. coli is considered transitory in the oral cavity, and responsible for initial yeast adherence to several surfaces ${ }^{24} P$. aeruginosa is an opportunistic pathogen that hardly ever causes disease in a healthy immune system; however, it exploits weaknesses of the body in order to establish an infection condition..$^{25}$ The yeasts selected were $C$. albicans, the fungi species most frequently found in denture biofilm, ${ }^{26}$ and $C$. glabrata, the second most prevalent Candida species in human beings, and frequently found in biofilm associated with denture stomatitis. ${ }^{27}$

The results showed that both concentrations of $\mathrm{NaOCl}$ applied in immersions of short duration (20 min) were effective, eliminating all microorganisms evaluated. Laboratory studies have reported on the antimicrobial action of $\mathrm{NaOCl}$ in higher concentrations and different immersion periods. Rudd et al. ${ }^{10}$ demonstrated that a $5.25 \%$ concentration and a 5 min immersion was an efficient disinfecting agent for complete dentures. A 1\% concentration has been effective in disinfecting acrylic resin specimens contaminated with strains of $S$. aureus, C. albicans,

Table. Mean values and standard deviation for species, in $\log 10(\mathrm{CFU}+1) / \mathrm{mL}$, average differences among the groups and Student's t-test values for independent samples.

\begin{tabular}{lcccc}
\hline Species & Group D (Control) & Group C (Castor oil 10\%) & Difference C-D (95\%Cl) & $p$-value \\
\hline B. subtilis & $1.45(0.85)$ & $0.00(0.00)$ & $-1.45\left(\mathrm{n} / \mathrm{a}^{\dagger}\right)$ & $\mathrm{n} / \mathrm{a}^{\dagger}$ \\
C. albicans & $2.68(0.39)$ & $1.66(0.68)$ & $-1.02(-1.55$ to -0.50$)$ & $0.001^{*}$ \\
C. glabrata & $5.11(0.22)$ & $1.13(1.16)$ & $-3.98(-4.81$ to -3.14$)$ & $<0.001^{*}$ \\
E. coli & $3.62(0.70)$ & $2.09(1.13)$ & $-1.54(-2.42$ to -0.65$)$ & $0.002^{*}$ \\
E. faecalis & $3.64(1.02)$ & $3.54(0.73)$ & $-0.10(-0.93$ to 0.73$)$ & $0.805^{\text {ns }}$ \\
P. aeruginosa & $6.24(0.36)$ & $4.44(0.78)$ & $-1.80(-2.37$ to - 1.23$)$ & $<0.001^{*}$ \\
S. aureus & $3.88(0.69)$ & $2.71(1.26)$ & $-1.17(-2.12$ to -0.21$)$ & $0.019^{*}$ \\
S. mutans & $5.97(0.82)$ & $3.70(0.81)$ & $-2.27(-3.04$ to - 1.51$)$ & $<0.001^{*}$ \\
\hline
\end{tabular}

† Test not performed, because all specimens in Group $C$ recorded 0 CFU.

ns no significant difference ( $p>0.05)$.

"significant difference $(p<0.05)$ 
S. mutans, P. aeruginosa, E. coli and B. subtilis, after 10-15 min of immersion. ${ }^{11,12,28}$ Other studies found that $1.5 \%$ concentrations for $20 \mathrm{~min}$ and $2 \%$ for $5 \mathrm{~min}$ have been effective against strains of C. albicans. ${ }^{28,29}$ However, it is important to consider the concentration used, because $\mathrm{NaOCl}$ may cause alterations in acrylic resin properties. ${ }^{7,8}$

A $0.5 \%$ concentration has proven effective against C. albicans, after 10 min of immersion. ${ }^{30}$ Our results showed the effectiveness of a $0.5 \%$ concentration against strains of $C$. glabrata and bacterial species, such as E. faecalis, S. mutans, S. aureus, P. aeruginosa, E. coli and B. subtilis.

The antimicrobial activity results for a $0.25 \%$ concentration are relevant, suggesting that $\mathrm{NaOCl}$ can be used in lower concentrations. Regarding the adverse effects, Paranhos et al. ${ }^{7}$ simulated overnight cleansing for $1 \frac{1}{2} \mathrm{yr}$ and found alterations in color properties and increased surface roughness of an acrylic resin, using a $0.5 \%$ concentration. However, the changes are unlikely to have any clinical significance. Thus, the results showed that both concentrations applied in short-term immersions ( $20 \mathrm{~min}$ ) may be useful as auxiliary hygiene agents in controlling complete denture biofilms. Nevertheless, studies on possible adverse effects are needed, since no results for a $0.25 \%$ concentration have been reported in the literature.

Just like the $\mathrm{NaOCl}$ solution, the castor oil solution showed complete B. subtilis elimination, whereas different disinfectants (glutaraldehyde and chlorhexidine) have not proven effective. ${ }^{11} B$. subtilis is an opportunistic microorganism, not showing sufficient adherent capacity when isolated, but promoting biofilm growth and adherence of other microorganisms. ${ }^{23}$ Therefore, its elimination is crucial to denture maintenance and oral health.

The castor oil solution was not effective against E. faecalis. Paranhos et al. ${ }^{2}$ revealed that the combined method (brushing followed by immersion) was similar to brushing alone, indicating only a minor effect of the chemical method on this microorganism. Orsi et al. ${ }^{12}$ noted that $E$. faecalis was the most resistant strain, promoting microbial growth on the internal surfaces of acrylic resin after immersion in chemical solutions. Castor oil solution has been found to provide antimicrobial action against other microorganisms (C. albicans, C. glabrata, S. aureus, E. coli, P. aeruginosa and $S$. mutans), suggesting the solution can be used against complete denture biofilm.

Evaluating the microorganisms individually was a limitation of this study, since antimicrobial activity was evaluated in relation to a simple biofilm. Therefore, further studies involving mixed biofilms should be performed to provide data for microbial interaction. Another aspect to be investigated concerns the possible adverse effects caused to denture materials; thus, the analysis of both products, i.e. hypochlorite and castor oil, must be conducted in the concentrations tested in the study, in order to establish the immersion protocol.

\section{Conclusion}

Immersion in sodium hypochlorite solutions $(0.25 \%$ and $0.5 \%$ ) is an efficacious method of eliminating pathogens present in denture biofilm. These solutions may prove useful for cleaning complete dentures. Castor oil solution provided moderate efficacy and had a varied effect on different species.

\section{Acknowledgements}

The authors wish to thank Professors Gilberto Orivaldo Chierice (Institute of Chemistry of São Carlos, Universidade de São Paulo - USP), for providing the castor oil solution, and Evandro Watanabe (School of Dentistry of Ribeirão Preto, Universidade de São Paulo, Brazil) for helping with the microbial analysis.

\section{References}

1. Paranhos HFO, Salles AES, Macedo LD, Silva-Lovato $\mathrm{CH}$, Pagnano VO, Watanabe E. Complete denture biofilm after brushing with specific denture paste, neutral soap and artificial saliva. Braz Dental J. 2013;24(1):47-52.
2. Paranhos HFO, Silva CHL, Souza RF, Cruz PC, Freitas KM, Ito IY, et al. Effect of three methods for cleaning dentures biofilms formed in vitro on acrylic resin. J Prosthodont 2009 Jul;18(5):427-431. 
3. Andrade IM, Andrade KM, Pisani MX, Silva-Lovato $\mathrm{CH}$, Souza RF, Paranhos HFO. Trial of an experimental castor oil solution for cleaning dentures. Braz Dent J. 2014 Jan-Feb;25(1):43-7.

4. Lima EM, Moura JS, Del Bel Cury AA, Garcia RC, Cury JA. Effect of enzymatic and $\mathrm{NaOCl}$ treatments on acrylic roughness and on biofilm accumulation. J Oral Rehabil. 2006 May;33(5):356-62.

5. Nikawa H, Hamada T, Yamashiro H, Kumagai H. A review of in vitro and in vivo methods to evaluate the efficacy of denture cleansers. Int J Prosthodont. 1999 Mar-Apr;12(2):153-9.

6. Souza RF, Paranhos HFO, Silva-Lovato CH, Abu-Naba'a L, Fedorowicz Z, Gurgan CA. Interventions for cleaning dentures in adults. Cochrane Database of Syst Rev. 2009 Oct 7;(4):CD007395.

7. Paranhos HFO, Peracini A, Pisani MX, Oliveira VC, Souza $\mathrm{RF}$, Silva-Lovato $\mathrm{CH}$. Color stability, surface roughness and flexural strength of an acrylic resin submitted to simulated overnight immersion in denture cleansers. Braz Dent J. 2013;24(2):152-6.

8. Davi LR, Felipucci DNB, Souza RF, Bezzon OL, Silva-Lovato $\mathrm{CH}$, Souza VOP, et al. Effect of denture cleansers on metal Ion release and surface roughness of denture base materials. Braz Dent J. 2012;23(4):387-93.

9. Felipucci DNB, Davi LR, Paranhos HFO, Bezzon OL, Silva RF, Pagnano VO. Effect of different cleansers on the surface of removable partial denture. Braz Dent J. 2011;22(5):392-7.

10. Rudd RW, Senia ES, McCleskey FK, Adams EDJr. Sterilization of complete dentures with sodium hypochlorite. J Prosthet Dent. 1984 Mar;51(3):318-21.

11. Silva FC, Kimpara ET, Mancini MN, Balducci I, Jorge AO, Koga-Ito CY. Effectiveness of six different disinfectants on removing five microbial species and effects on the topographic characteristics of acrylic resin. J Prosthodont. 2008 Dec;17(8):627-33.

12. Orsi IA, Junior AG, Villabona CA, Fernandes FH, Ito IY. Evaluation of the efficacy of chemical disinfectants for disinfection of heat-polymerised acrylic resin. Gerodontology. 2011 Dec;28(4):253-7.

13. Ferreira CM, Bonifacio KC, Froner IC, Ito IY. Evaluation of the antimicrobial activity of three irrigation solutions in teeth with pulpal necrosis. Braz Dent J. 1999;10(1):15-21.

14. Ferreira CM, Rosa OPS, Torres AS, Ferreira FBA, Bernardinelli $\mathrm{N}$. Activity of endodontic antibacterial agents against selected anaerobic bacteria. Braz Dent J. 2002;13(2):118-22.

15. Pisani MX, Silva-Lovato $\mathrm{CH}$, Paranhos HFO, Souza RF, Macedo AP. The effect of experimental denture cleanser solution Ricinus communis on acrylic resin properties. Mater Res. 2010;13(3):369-73.

16. Pisani MX, Macedo AP, Paranhos HFO, Silva-Lovato $\mathrm{CH}$. Effect of experimental Ricinus communis solution for denture cleaning on the properties of acrylic resin teeth. Braz Dent J. 2012;23(1):15-21.

17. Pinelli LAP, Montandon AAB, Corbi SCT, Moraes TA. Ricinus communis treatment of denture stomatitis in institutionalised elderly. J Oral Rehabil. 2013 May;40(5):375-80.

18. Leite VMF, Pinheiro JB, Pisani MX, Watanabe E, Souza RF, Paranhos HFO, et al. In vitro antimicrobial activity of an experimental dentifrice based on Ricinus communis. Braz Dent J. 2014;25(3):191-196.

19. Silva MM, Vergani CE, Giampaolo ET, Neppelenbroek, KH, Spolidorio DM, Machado AL. Effectiveness of microwave irradiation on the disinfection of complete dentures. Int $\mathrm{J}$ Prosthodont. 2006 May-Jun;19(3):288-93.

20. Cole EC, Robinson R. Test methodology for evaluation of germicides. In: Ascenzi JM. Handbook of disinfectants and antiseptics. New York: Marcel Dekker Inc. 1996; 1-13.

21. Smith AJ, Jackson MS, Bagg J. The ecology of Staphylococcus species in the oral cavity. J Med Microbiol. 2001 Nov;50(11):940-6.

22. Paradella TC, Koga-Ito CY, Jorge AOC. Enterococcus faecalis: considerações clínicas e microbiológicas. Rev Odontol Unesp. 2007;36(2):163-6.

23. Tam NK, Uyem NQ, Hong HA, Duc LH, Hoa TT, Serra $\mathrm{CR}$, et al. The intestinal life cycle of bacillus subtilis and close relatives. J Bacteriol. 2006 Apr;188(7):2692-700.

24. Samaranayake LP, Lamb AB, Lamey PJ, MacFarlane TW. Oral carriage of Candida species and coliforms in patients with burning mouth syndrome. J Oral Pathol Med. 1989 Apr;18(4):233-5.

25. Lyczak JB, Cannon CL, Pier GB. Establishment of Pseudomonas aeruginosa infection: lessons from a versatile opportunist. Microbes Infect. 2000 Jul; 2(9):1051-60.

26. Salerno C, Pascale M, Contaldo M, Esposito V, Busciolano M, Milillo L, et al. Candida-associated denture stomatitis. Med Oral Patol Oral Cir Bucal. 2011 Mar;16(2):e139-43.

27. Coco BJ, Bagg J, Cross LJ, Jose A, Cross J, Ramage G. Mixed Candida albicans and Candida glabrata populations associated with the pathogenesis stomatitis. Oral Microbiol Immunol. 2008 Oct;23(5):377-83.

28. Silva PMB, Acosta EJTR, Pinto LR, Graeff M, Spolidorio DM, Almeida RS, et al. Microscopical analysis of Candida albicans biofilms on heat-polymerised acrylic resin after chlorhexidine gluconate and sodium hypochlorite treatments. Mycoses. 2011 Nov;54(6):e712-7.

29. Jose A, Coco BJ, Milligan S, Young B, Lappin DF, Bagg J, et al. Reducing the incidence of denture stomatitis: Are denture cleansers sufficient? J Prosthodont. 2010 Jun;19(4):252-7.

30. Montagner H, Montagner F, Braun KO, Peres PEC, Gomes BPFA. In vitro antifungal action of different substances over microwaved-cured acrylic resins. J Appl Oral Sci. 2009 Sep-Oct;17(5):432-5. 